\title{
Solvability and Lyapunov stability of a two-component system of generalized Poisson-Nernst-Planck equations
}

\author{
Victor A. Kovtunenko and Anna V. Zubkova
}

\begin{abstract}
A time-dependent Poisson-Nernst-Planck system of nonlinear partial differential equations of a mixed parabolic-elliptic type is considered. The generalized model is supplemented by a positivity and volume constraints, by dual entropy variables, and by nonlinear boundary conditions. The existence theorem supported by a-priori estimates, the Lyapunov stability of the solution, and its uniqueness in a special case are proved.
\end{abstract}

Mathematics Subject Classification (2000). Primary 35D05; Secondary 35M10, $37 \mathrm{~B} 25,82 \mathrm{C} 24$.

Keywords. Generalized Poisson-Nernst-Planck model, nonlinear parabolicelliptic system, nonlinear boundary conditions, existence and uniqueness, Lyapunov stability.

\section{Introduction}

In the paper we provide existence, uniqueness, and stability of a weak solution for a mixed parabolic-elliptic system of nonlinear Poisson-Nernst-Planck (PNP) equations.

The PNP system has many applications in bio- and electro-chemistry. The modeling issues are considered in $[5,9,10,19]$ for multi-component coupled PNP system, in particular, its application to battery models in $[2,15]$. For the methods used to prove existence of weak solutions, we refer to $[6,11,16]$ in the dynamic case, and to $[3,8]$ in the stationary case. The another important task is homogenization of these models, see the related works by $[1,7,18]$.

We consider a specific time-dependent PNP system consisting of two nonlinear parabolic equations coupled with a nonlinear elliptic equation. It describes

This work was completed with the support of the Austrian Science Fund (FWF) Project P26147N26: "Object identification problems: numerical analysis" (PION). 
electrokinetic phenomena in a two-component mixture of charged species. The model contains also dual entropy variables, which are redundant and came into our consideration from physical laws.

Below we point out the main specialities of our model and difficulties of its mathematical analysis in the current paper.

First, we consider inhomogeneous, moreover, nonlinear boundary conditions. In comparison, in the literature, see e.g. [11, 16], boundary conditions are assumed to be homogeneous. The nonlinearity at the boundary claims supplementary difficulties of analysis.

Another difficulty is the presence of unilateral, positivity and volume balance constraints, which do not allow to prove existence of the solution via classical methods. For this task, two formulations, namely, the complete formulation with constraints and the reduced one without constraints are employed. The equivalence of two formulations under reasonable assumptions will be shown. As the result, we prove the existence theorem, equipped with a-priori estimates, and the Lyapunov stability of a weak solution. Because of the nonlinearity of the system we cannot prove uniqueness of the solution in a general case. Nevertheless, we state the uniqueness theorem in a special case with additional assumptions on the solution regularity and for small boundary terms. The idea of the proof is similar to $[11,17]$.

In order to state well-posedness and stability of this problem, we use variational methods and derive a-priori estimates. Then the existence follows from the Tikhonov-Schauder fixed point theorem. For Lyapunov stability we define the Lyapunov entropy function following [4], and we prove the dissipation inequality under additional assumptions on the boundary terms.

The structure of the paper is the following. In Section 2 we give, first, the complete, and then the reduced formulations of the problem in the strong and in the weak forms. In Section 3 there are proved the existence theorems, based on three auxillary lemmas. At the end we establish the uniqueness theorem and stability of the solution in the sense of Lyapunov.

\section{Strong and weak formulations of the problem}

We begin with a description of the geometry.

Let $\Omega$ be a bounded domain in $\mathbb{R}^{d}, d=\{1,2,3\}$, with the Lipschitz boundary $\partial \Omega=\bar{\Gamma}_{N} \cup \bar{\Gamma}_{D}$ consisted of two disjoint parts such that $\Gamma_{N} \cap \Gamma_{D}=\emptyset, \Gamma_{D} \neq \emptyset$, and let $\nu=\left(\nu_{1}, \ldots, \nu_{d}\right)$ be the unit normal vector on $\Gamma_{N}$ outward to $\Omega$. For arbitrarily fixed final time $T>0$ we introduce the cylinder $Q_{T}:=(0, T) \times \Omega$.

\subsection{The strong formulation of the complete problem}

For $(t, x) \in Q_{T}$ we consider the unknown scalar function $\varphi(t, x)$ and the vectorfunction $\mathbf{c}:=\left(c_{1}, c_{2}\right)$, consisted of two components $c_{1}(t, x)$ and $c_{2}(t, x)$. These functions imply a solution of the Poisson-Nernst-Planck system of the mixed 


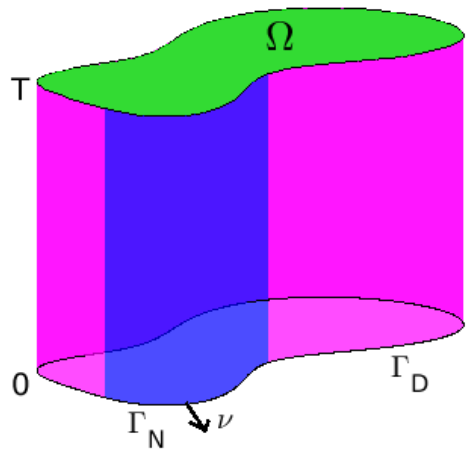

Figure 1. An example geometric domain

parabolic-elliptic type:

$$
\begin{gathered}
\frac{\partial c_{i}}{\partial t}=\operatorname{div}\left(c_{i} \nabla \mu_{i}\right), \quad i=1,2, \quad \text { in } \quad(0, T) \times \Omega ; \\
-\Delta \varphi=\frac{1}{2}\left(c_{1}-c_{2}\right) \quad \text { in } \quad(0, T) \times \Omega ; \\
\nabla p=-\frac{1}{2}\left(c_{1}-c_{2}\right) \nabla \varphi \quad \text { in } \quad(0, T) \times \Omega ; \\
\mu_{i}=\ln c_{i}+p+z_{i} \varphi, \quad i=1,2, \quad \text { in } \quad(0, T) \times \Omega .
\end{gathered}
$$

The system (2.1) is written in the dimensionless form. Here $c_{1}$ and $c_{2}$ associate the concentrations of two species with the charges $z_{1}=1$ and $z_{2}=-1$, and $\varphi$ stands for the overall electrostatic potential in a pore medium. We note that the equations (2.1a), after substitution here $\nabla p$ from (2.1c) and $\nabla \mu_{i}$ from (2.1d) have the entire form (2.12). They are coupled with (2.1b) by means of the nonlinear terms in the right-hand sides. Equations (2.1c) and (2.1d) are redundant and serve to determine the dual entropy variables $\mu_{1}, \mu_{2}$, which are associated to the quasiFermi (electrochemical) potentials, and $p$ standing for the pressure.

Next, the system (2.1) will be endowed with appropriate boundary and initial conditions. We suggest nonlinear Neumann conditions:

$$
\left(c_{i} \nabla \mu_{i}\right)^{\top} \nu=g_{i}(\mathbf{c}, \varphi), \quad i=1,2, \quad \text { on } \quad(0, T) \times \Gamma_{N},
$$

where the nonlinear functions $g_{1}(\mathbf{c}, \varphi)$ and $g_{2}(\mathbf{c}, \varphi)$ are motivated by boundary reactions in electrochemical mixtures and will be specified in Section 2.2; and

$$
\begin{array}{ll}
\text { Robin condition: } & \nabla \varphi^{\top} \nu+\varphi=g \text { on }(0, T) \times \Gamma_{N} ; \\
\text { Dirichlet conditions: } & \varphi=0, \quad c_{1}=c_{2}=1 \quad \text { on }(0, T) \times \Gamma_{D} .
\end{array}
$$

In (2.3) the function $g \in L^{\infty}\left(0, T ; L^{2}\left(\Gamma_{N}\right)\right)$ is given, and (2.4) implies non-dimensional boundary data, where we normalize the positive concentrations $c_{1}$ and $c_{2}$ to one. 
The standard initial conditions are supposed:

$$
c_{1}(0, \cdot)=c_{1}^{0}, \quad c_{2}(0, \cdot)=c_{2}^{0} \quad \text { as } \quad t=0,
$$

where $c_{1}^{0}$ and $c_{2}^{0}$ are given functions in $L^{2}(\Omega)$.

Additionally to the boundary and initial conditions, the system is subject to the volume balance constraint:

$$
c_{1}+c_{2}=2 \text { in }(0, T) \times \Omega,
$$

and the positivity constraint:

$$
c_{1}>0, \quad c_{2}>0 \quad \text { in }(0, T) \times \Omega .
$$

The value " 2 " in (2.6a) is due to the compatibility with the Dirichlet data (2.4), such that $c_{1}+c_{2}=2$ at $(0, T) \times \Gamma_{D}$. These constraints appear naturally from the point of view of physical consistency, but they bring the principle difficulty in the mathematical investigation of this problem.

\subsection{Assumptions on the data}

The following assumptions on the boundary terms $g_{1}(\mathbf{c}, \varphi), g_{2}(\mathbf{c}, \varphi) \in L^{2}((0, T) \times$

$\left.\Gamma_{N}\right)$ will be required further. Namely, the following three conditions are assumed: continuity and the growth condition with uniformly bounded $0 \leqslant G(\mathbf{c}) \leqslant 1$ :

$$
\int_{0}^{T} \int_{\Gamma_{N}}\left|g_{i}(\mathbf{c}, \varphi)\right|^{2} d x d t \leqslant\left(\beta_{1}+\beta_{2}\|\varphi\|_{L^{2}\left(0, T ; H^{1}(\Omega)\right)}^{2}\right) G(\mathbf{c}), \quad i=1,2
$$

the mass conservation: $g_{1}(\mathbf{c}, \varphi)+g_{2}(\mathbf{c}, \varphi)=0 \quad$ on $\quad(0, T) \times \Gamma_{N}$;

the positive production rate:

$g_{1}(\mathbf{c}, \varphi) c_{1}^{-}=g_{2}(\mathbf{c}, \varphi) c_{2}^{-}=0 \quad$ on $\quad(0, T) \times \Gamma_{N}, \quad$ for all $c_{1}, c_{2}$.

In $(2.7 \mathrm{c})$ the partition in the positive and the negative parts is defined by

$$
c_{i}^{+}:=\max \left\{0, c_{i}\right\}, \quad c_{i}^{-}:=-\min \left\{0, c_{i}\right\}
$$

such that

$$
c_{i}=c_{i}^{+}-c_{i}^{-}, \quad c_{i}^{+}, c_{i}^{-} \geqslant 0, \quad c_{i}^{+} \cdot c_{i}^{-}=0, \quad i=1,2 .
$$

We note that the condition (2.7c) appears as a consequence of tangential conditions from quasi-linear ODE [6, Chapter 3].

Example. The functions $g_{1}(\mathbf{c}, \varphi)=\frac{c_{1}^{+} c_{2}^{+}}{\left(c_{1}^{+}+c_{2}^{+}\right)^{2}}$ and $g_{2}(\mathbf{c}, \varphi)=-\frac{c_{1}^{+} c_{2}^{+}}{\left(c_{1}^{+}+c_{2}^{+}\right)^{2}}$ for $c_{1}+c_{2}=2$ fulfill all assumptions $(2.7)$.

The initial data in (2.5) should also satisfy the constraints (2.6):

$$
\begin{gathered}
c_{1}^{0}+c_{2}^{0}=2 \quad \text { in } \quad(0, T) \times \Omega ; \\
c_{1}^{0}>0, \quad c_{2}^{0}>0 \quad \text { in } \quad(0, T) \times \Omega .
\end{gathered}
$$

The assumptions (2.7)-(2.9) will be employed for the existence theorems in Section 3.1. 


\subsection{The weak formulation of the complete problem}

We will endow the problem (2.1)-(2.5) with the weak formulation:

Find $c_{1}, c_{2}, \varphi, \mu_{1}, \mu_{2}, p$ such that

$$
\begin{gathered}
c_{1}, c_{2} \in L^{\infty}\left(0, T ; L^{2}(\Omega)\right) \cap L^{2}\left(0, T ; H^{1}(\Omega)\right), \\
\varphi, p \in L^{\infty}\left(0, T ; H^{1}(\Omega)\right), \\
c_{1} \nabla \mu_{1}, c_{2} \nabla \mu_{2} \in L^{2}\left(Q_{T}\right),
\end{gathered}
$$

which satisfy the Dirichlet boundary conditions (2.4), the initial conditions (2.5), the volume and the positivity constraints (2.6), as well as fulfill two dynamic and one quasi-stationary variational equations for a time $\tau \in(0, T)$ :

$$
\begin{gathered}
\left.\int_{\Omega} c_{i} \bar{c}_{i} d x\right|_{0} ^{\tau}-\int_{Q \tau} c_{i} \frac{\partial \bar{c}_{i}}{\partial t} d x d t+\int_{Q \tau}\left(c_{i} \nabla \mu_{i}\right)^{\top} \nabla \bar{c}_{i} d x d t \\
=\int_{0}^{\tau} \int_{\Gamma_{N}} g_{i}(\mathbf{c}, \varphi) \bar{c}_{i} d S_{x} d t, \quad i=1,2, \\
\int_{\Omega}\left(\nabla \varphi^{\top} \nabla \bar{\varphi}-\frac{1}{2}\left(c_{1}-c_{2}\right) \bar{\varphi}\right) d x+\int_{\Gamma_{N}} \varphi \bar{\varphi} d S_{x}=\int_{\Gamma_{N}} g \bar{\varphi} d S_{x},
\end{gathered}
$$

for all test functions $\bar{c}_{1}, \bar{c}_{2} \in H^{1}\left(0, T ; L^{2}(\Omega)\right) \cap L^{2}\left(0, T ; H^{1}(\Omega)\right)$ and $\bar{\varphi} \in H^{1}(\Omega)$, such that $\bar{c}_{1}=\bar{c}_{2}=\bar{\varphi}=0$ on $\Gamma_{D}$.

The system (2.11) is completed with two identities (2.1c) and (2.1d) describing the dual entropy variables $p$ and $\mu_{1}, \mu_{2}$ almost everywhere in $(0, T) \times \Omega$.

The main difficulty is the presence of the constraints (2.6) in the system. Indeed, they cannot be guaranteed from the classic solution theory. By this reason, in Section 2.4 we suggest a reduced formulation of the problem. In Lemma 3.4 in Section 3 the equivalence of these two formulations under reasonable assumptions will be proved, in this way providing solvability of the complete system.

\subsection{The reduced system of equations}

The reduced formulation excludes $\mu_{1}, \mu_{2}$ and $p$ as well as the constraints (2.6).

First, with the help of (2.1c) and (2.1d) we exclude the dual entropy variables from (2.1a) and write it in the equivalent form:

$$
\frac{\partial c_{i}}{\partial t}=\operatorname{div}\left(\nabla c_{i}+z_{i} c_{1} c_{2} \nabla \varphi\right), \quad i=1,2, \quad \text { in } \quad(0, T) \times \Omega .
$$

Second, we exclude constraints and restate the equations (2.12) and (2.1b) as

$$
\begin{gathered}
\frac{\partial c_{i}}{\partial t}=\operatorname{div}\left(\nabla c_{i}+\Upsilon_{i}\left(\mathbf{c}^{+}\right) \nabla \varphi\right), \quad i=1,2, \quad \text { in } \quad(0, T) \times \Omega ; \\
-\Delta \varphi=\Upsilon\left(\mathbf{c}^{+}\right) \quad \text { in } \quad(0, T) \times \Omega ;
\end{gathered}
$$

under the corresponding Neumann-Robin boundary conditions

$$
\begin{gathered}
\left(\nabla c_{i}+\Upsilon_{i}\left(\mathbf{c}^{+}\right) \nabla \varphi\right)^{\top} \nu=g_{i}(\mathbf{c}, \varphi), \quad i=1,2, \quad \text { on } \quad(0, T) \times \Gamma_{N} \\
\nabla \varphi^{\top} \nu+\varphi=g \quad \text { on } \quad(0, T) \times \Gamma_{N} ;
\end{gathered}
$$


the Dirichlet boundary conditions (2.4), and the initial conditions (2.5). In the equations (2.13) and (2.14a) the following notations are used:

$$
\Upsilon_{i}\left(\mathbf{c}^{+}\right):=\frac{4 z_{i} c_{1}^{+} c_{2}^{+}}{\left(c_{1}^{+}+c_{2}^{+}\right)^{2}}, \quad \Upsilon\left(\mathbf{c}^{+}\right):=\frac{c_{1}^{+}-c_{2}^{+}}{c_{1}^{+}+c_{2}^{+}},
$$

recalling the definition of the positive part given in (2.8).

The reason behind (2.15) is that, if the constraints (2.6) hold, then (2.15) turns into expressions

$$
\Upsilon_{1}\left(\mathbf{c}^{+}\right)=c_{1} c_{2}, \quad \Upsilon_{2}\left(\mathbf{c}^{+}\right)=-c_{1} c_{2}, \quad \Upsilon\left(\mathbf{c}^{+}\right)=\frac{1}{2}\left(c_{1}-c_{2}\right),
$$

which enter the right-hand sides of (2.12) and (2.1b).

For the reduced problem we give the weak formulation, which is similar to (2.10) and (2.11): Find $c_{1}, c_{2}, \varphi$ such that

$$
\begin{gathered}
c_{1}, c_{2} \in L^{\infty}\left(0, T ; L^{2}(\Omega)\right) \cap L^{2}\left(0, T ; H^{1}(\Omega)\right), \\
\varphi \in L^{\infty}\left(0, T ; H^{1}(\Omega)\right),
\end{gathered}
$$

satisfying the Dirichlet boundary conditions (2.4), the initial conditions (2.5), and the following variational equations:

$$
\begin{gathered}
\left.\int_{\Omega} c_{i} \bar{c}_{i} d x\right|_{0} ^{\tau}-\int_{Q \tau} c_{i} \frac{\partial \bar{c}_{i}}{\partial t} d x d t+\int_{Q \tau}\left(\nabla c_{i}+\Upsilon_{i}\left(\mathbf{c}^{+}\right) \nabla \varphi\right)^{\top} \nabla \bar{c}_{i} d x d t \\
=\int_{0}^{\tau} \int_{\Gamma_{N}} g_{i}(\mathbf{c}, \varphi) \bar{c}_{i} d S_{x} d t \\
\int_{\Omega}\left(\nabla \varphi^{\top} \nabla \bar{\varphi}-\Upsilon\left(\mathbf{c}^{+}\right) \bar{\varphi}\right) d x+\int_{\Gamma_{N}} \varphi \bar{\varphi} d S_{x}=\int_{\Gamma_{N}} g \bar{\varphi} d S_{x},
\end{gathered}
$$

for all test functions $\bar{c}_{1}, \bar{c}_{2} \in H^{1}\left(0, T ; L^{2}(\Omega)\right) \cap L^{2}\left(0, T ; H^{1}(\Omega)\right)$ and $\bar{\varphi} \in H^{1}(\Omega)$, such that $\bar{c}_{1}=\bar{c}_{2}=\bar{\varphi}=0$ on $\Gamma_{D}$.

Based on the reduced formulation, in the next section we will provide wellposedness of the underlying problem.

\section{Well-posedness of the problem}

We start with the following preliminaries of functional analysis.

Trace theorem. Let $u \in H^{1}(\Omega)$, then

$$
k_{0}\|u\|_{H^{1}(\Omega)}^{2} \leqslant\|u\|_{H^{1 / 2}(\partial \Omega)}^{2} \leqslant K_{0}\|u\|_{H^{1}(\Omega)}^{2},
$$

where $0<k_{0} \leqslant K_{0}$, and the standard norms are:

$$
\begin{aligned}
& \|u\|_{H^{1 / 2}(\partial \Omega)}^{2}=\|u\|_{L^{2}(\partial \Omega)}^{2}+\int_{\partial \Omega} \int_{\partial \Omega} \frac{|u(x)-u(y)|^{2}}{|x-y|^{d}} d x d y, \\
& \|u\|_{H^{1}(\Omega)}^{2}=\|u\|_{L^{2}(\Omega)}^{2}+\|\nabla u\|_{L^{2}(\Omega)^{d}}^{2} .
\end{aligned}
$$


Poincaré inequality. Let $u \in H^{1}(\Omega), u=0$ on $\Gamma_{D}$ or $\int_{\Omega} u d x=0$. Then

$$
\|u\|_{L^{2}(\Omega)}^{2} \leqslant K_{P}\|\nabla u\|_{L^{2}(\Omega)^{d}}^{2}
$$

where $K_{P}>0$ depends on $\Omega$ and $d$. The Poincaré inequality implies that

$$
\frac{1}{1+K_{P}}\|u\|_{H^{1}(\Omega)}^{2} \leqslant\|\nabla u\|_{L^{2}(\Omega)^{d}}^{2} \leqslant\|u\|_{H^{1}(\Omega)}^{2} .
$$

\subsection{The existence theorems}

In this section we state two existence theorems and three auxillary lemmas. At first, the absence of the constraints allows us to prove the existence of the solution of the reduced problem based on a fixed-point iteration. Thereafter, we show that this solution satisfies the volume balance and positivity conditions (2.6) under reasonable assumptions. In this case, this fact guarantees the equivalence of two formulations. The main result of this section is well-posedness of the generalized Poisson-Nernst-Planck system in the complete formulation.

Theorem 3.1 (Existence of a weak solution of the reduced problem). Let the continuity and the growth conditions (2.7a) hold. Then there exists a weak solution (2.17) of the reduced problem (2.18) under the boundary (2.4) and initial (2.5) conditions.

Proof. The proof is based on the Schauder-Tikhonov fixed point theorem.

First, we choose an initialization

$$
c_{1}^{(0)}, c_{2}^{(0)} \in L^{\infty}\left(0, T ; L^{2}(\Omega)\right) \cap L^{2}\left(0, T ; H^{1}(\Omega)\right)
$$

such that the following relations hold:

$$
\begin{aligned}
& c_{1}^{(0)}=c_{2}^{(0)}=1 \quad \text { on } \quad(0, T) \times \Gamma_{D}, \\
& c_{1}^{(0)}+c_{2}^{(0)}=2, \quad c_{1}^{(0)}>0, \quad c_{2}^{(0)}>0 \quad \text { in } \quad(0, T) \times \Omega .
\end{aligned}
$$

For a fixed time $\tau \in(0, T)$, we consider the sequence $\left\{\left(c_{1}^{(k+1)}, c_{2}^{(k+1)}, \varphi^{(k+1)}\right)\right\}$, $k \in \mathbb{N}_{0}$, which is determined as an iterative solution of the linearized equations (2.18) starting with $k=0$ :

$$
\begin{aligned}
\int_{\Omega}\left(\nabla \varphi^{(k+1)}\right)^{\top} \nabla \bar{\varphi} d x+ & \int_{\Gamma_{N}} \varphi^{(k+1)} \bar{\varphi} d S_{x} \\
& =\int_{\Gamma_{N}} g \bar{\varphi} d S_{x}+\int_{\Omega} \Upsilon\left(\left(\mathbf{c}^{(k)}\right)^{+}\right) \bar{\varphi} d x \\
\int_{Q_{\tau}} \frac{\partial c_{i}^{(k+1)}}{\partial t} \bar{c}_{i} d x d t+ & \int_{Q_{\tau}}\left(\nabla c_{i}^{(k+1)}\right)^{\top} \nabla \bar{c}_{i} d x d t=\int_{0}^{\tau} \int_{\Gamma_{N}} g_{i}^{(k)} \bar{c}_{i} d S_{x} d t \\
& -\int_{Q_{\tau}} \Upsilon_{i}\left(\left(\mathbf{c}^{(k)}\right)^{+}\right)\left(\nabla \varphi^{(k+1)}\right)^{\top} \nabla \bar{c}_{i} d x d t, \quad i=1,2,
\end{aligned}
$$

where $g_{i}^{(k)}:=g_{i}\left(\mathbf{c}^{(k)}, \varphi^{(k+1)}\right)$ for short. We emphasize that the equation (3.4) has to be solved at the first step, and its solution $\varphi^{(k+1)}$ enters the right-hand 
side of the equations (3.5) to be solved at the second step. The existence of the unique solution of this system follows from the standard linear theory of elliptic and parabolic equations [14].

Second, we want to obtain subsequent estimations for $\varphi^{(k+1)}$ and $\mathbf{c}^{(k+1)}$, which are independent of the iteration number $k$.

Estimation for $\varphi^{(k+1)}$. Let us choose the test function $\bar{\varphi}=\varphi^{(k+1)}$ in the equation (3.4) and estimate the following integral expression:

$$
\begin{aligned}
I_{\varphi}^{(k+1)}:=\int_{\Omega}\left|\nabla \varphi^{(k+1)}\right|^{2} d x & +\int_{\Gamma_{N}}\left|\varphi^{(k+1)}\right|^{2} d S_{x} \\
& =\int_{\Gamma_{N}} g \varphi^{(k+1)} d S_{x}+\int_{\Omega} \Upsilon\left(\left(\mathbf{c}^{(k)}\right)^{+}\right) \varphi^{(k+1)} d x .
\end{aligned}
$$

Initially we estimate $I_{\varphi}^{(k+1)}$ from above and, thereafter, from below by the norm of $\varphi^{(k+1)}$.

We note that $-1<\Upsilon\left(\left(\mathbf{c}^{(k)}\right)^{+}\right)<1$ is bounded uniformly, since its summands in (2.15) satisfy:

$$
0<\frac{\left(c_{1}^{(k)}\right)^{+}}{\left(c_{1}^{(k)}\right)^{+}+\left(c_{2}^{(k)}\right)^{+}}<1, \quad-1<-\frac{\left(c_{2}^{(k)}\right)^{+}}{\left(c_{1}^{(k)}\right)^{+}+\left(c_{2}^{(k)}\right)^{+}}<0 .
$$

Therefore, using Young's inequality with an arbitrary $\beta_{3}>0$ and Poincaré inequality (3.2), it follows boundedness of the second term in the right-hand side of (3.6):

$$
\begin{aligned}
& \left|\int_{\Omega} \Upsilon\left(\left(\mathbf{c}^{(k)}\right)^{+}\right) \varphi^{(k+1)} d x\right| \leqslant \int_{\Omega}\left|\varphi^{(k+1)}\right| d x \\
& \quad \leqslant \frac{1}{2 \beta_{3}} \int_{\Omega} d x+\frac{\beta_{3}}{2} \int_{\Omega}\left|\varphi^{(k+1)}\right|^{2} d x \leqslant \frac{1}{2 \beta_{3}}|\Omega|+\frac{\beta_{3} K_{P}}{2} \int_{\Omega}\left|\nabla \varphi^{(k+1)}\right|^{2} d x
\end{aligned}
$$

where $|\Omega|$ denotes the Hausdorff measure of $\Omega$ in $\mathbb{R}^{d}$. Now we estimate the first term in the right-hand side of (3.6) using again the Young's inequality with an arbitrary constant $\beta_{4}>0$ :

$$
\left|\int_{\Gamma_{N}} g \varphi^{(k+1)} d S_{x}\right| \leqslant \frac{1}{2 \beta_{4}} \int_{\Gamma_{N}} g^{2} d S_{x}+\frac{\beta_{4}}{2} \int_{\Gamma_{N}}\left|\varphi^{(k+1)}\right|^{2} d S_{x} .
$$

Therefore, using (3.7) and (3.8), the upper bound in (3.6) holds:

$$
\begin{aligned}
I_{\varphi}^{(k+1)} \leqslant \frac{1}{2 \beta_{3}}|\Omega|+\frac{1}{2 \beta_{4}}\|g\|_{L^{2}\left(\Gamma_{N}\right)}^{2} & \\
& +\frac{\beta_{3} K_{P}}{2}\left\|\varphi^{(k+1)}\right\|_{H^{1}(\Omega)}^{2}+\frac{\beta_{4}}{2}\left\|\varphi^{(k+1)}\right\|_{L^{2}\left(\Gamma_{N}\right)}^{2} .
\end{aligned}
$$

The lower bound of the integral $I_{\varphi}^{(k+1)}$ :

$$
\frac{1}{1+K_{P}}\left\|\varphi^{(k+1)}\right\|_{H^{1}(\Omega)}^{2}+\left\|\varphi^{(k+1)}\right\|_{L^{2}\left(\Gamma_{N}\right)}^{2} \leqslant I_{\varphi}^{(k+1)}
$$


follows from the consequence of (3.3) and the Poincaré inequality (3.2).

Collecting together (3.9) and (3.10) we estimate $\varphi^{(k+1)}$ :

$$
k_{1}\left(\left\|\varphi^{(k+1)}\right\|_{H^{1}(\Omega)}^{2}+\left\|\varphi^{(k+1)}\right\|_{L^{2}\left(\Gamma_{N}\right)}^{2}\right) \leqslant \frac{1}{2 \beta_{3}}|\Omega|+\frac{1}{2 \beta_{4}} \int_{\Gamma_{N}} g^{2} d S_{x},
$$

where $k_{1}:=\min \left\{\frac{1}{1+K_{P}}-\frac{\beta_{3} K_{P}}{2}, 1-\frac{\beta_{4}}{2}\right\}$. That means that $k_{1}>0$ for $\beta_{3}, \beta_{4}$ chosen sufficiently small and there exists a constant $K_{2}(\tau)>0$, such that

$$
\begin{aligned}
\left\|\varphi^{(k+1)}\right\|_{H^{1}(\Omega)}^{2}+\left\|\varphi^{(k+1)}\right\|_{L^{2}\left(\Gamma_{N}\right)}^{2} \leqslant K_{2}(\tau) & \\
& :=\frac{1}{2 k_{1}}\left(\frac{1}{\beta_{3}}|\Omega|+\frac{1}{\beta_{4}}\|g\|_{L^{2}\left(\Gamma_{N}\right)}^{2}\right),
\end{aligned}
$$

for all indexes $k \in \mathbb{N}_{0}$. Taking the supremum over $\tau \in(0, T)$ in (3.11), we obtain the resulting estimate for $\varphi^{(k+1)}$ :

$$
\left\|\varphi^{(k+1)}\right\|_{L^{\infty}\left(0, T ; H^{1}(\Omega)\right)}^{2}+\left\|\varphi^{(k+1)}\right\|_{L^{\infty}\left(0, T ; L^{2}\left(\Gamma_{N}\right)\right)}^{2} \leqslant K_{\varphi}:=\sup _{\tau \in(0, T)} K_{2}(\tau) .
$$

Estimation for $\mathbf{c}^{(k+1)}$. Let us choose the test functions $\bar{c}_{i}=c_{i}^{(k+1)}-1$ for $i=1,2$ in (3.5), which are zero at $(0, T) \times \Gamma_{D}$. Using the identities $\frac{\partial c_{i}^{(k+1)}}{\partial t}=\frac{\partial\left(c_{i}^{(k+1)}-1\right)}{\partial t}$ and $\nabla c_{i}^{(k+1)}=\nabla\left(c_{i}^{(k+1)}-1\right)$, this yields:

$$
\begin{array}{r}
\frac{1}{2} \int_{Q_{\tau}} \frac{\partial}{\partial t}\left(c_{i}^{(k+1)}-1\right)^{2} d x d t+\int_{Q_{\tau}}\left|\nabla c_{i}^{(k+1)}\right|^{2} d x d t=\int_{0}^{\tau} \int_{\Gamma_{N}} g_{i}^{(k)}\left(c_{i}^{(k+1)}-1\right) d S_{x} d t \\
-\int_{Q_{\tau}} \Upsilon_{i}\left(\left(\mathbf{c}^{(k)}\right)^{+}\right)\left(\nabla \varphi^{(k+1)}\right)^{\top} \nabla c_{i}^{(k+1)} d x d t, \quad i=1,2 .
\end{array}
$$

The right-hand side of (3.13) will be estimated by using the assumption of the theorem and the previous result for $\varphi^{(k+1)}$.

We start by noting that the expression $\Upsilon_{i}\left(\left(\mathbf{c}^{(k)}\right)^{+}\right)$is bounded uniformly:

$$
\left|\Upsilon_{i}\left(\left(\mathbf{c}^{(k)}\right)^{+}\right)\right|=\left|4 z_{i} \frac{\left(c_{1}^{(k)}\right)^{+}}{\left(\left(c_{1}^{(k)}\right)^{+}+\left(c_{2}^{(k)}\right)^{+}\right)} \frac{\left(c_{2}^{(k)}\right)^{+}}{\left(\left(c_{1}^{(k)}\right)^{+}+\left(c_{2}^{(k)}\right)^{+}\right)}\right|<4, \quad i=1,2 .
$$

Using Young's inequality with the constants $\beta_{5}>0$ and $\beta_{6}>0$, Poincaré inequality (3.2) and the trace theorem (3.1), the estimates of the terms in the right-hand side of (3.13) follow:

$$
\begin{gathered}
\left|\int_{Q_{\tau}} \Upsilon_{i}\left(\left(\mathbf{c}^{(k)}\right)^{+}\right)\left(\nabla \varphi^{(k+1)}\right)^{\top} \nabla c_{i}^{(k+1)} d x d t\right|<\left|\int_{Q_{\tau}} 4\left(\nabla \varphi^{(k+1)}\right)^{\top} \nabla c_{i}^{(k+1)} d x d t\right| \\
\leqslant \frac{2}{\beta_{5}} \int_{Q_{\tau}}\left|\nabla \varphi^{(k+1)}\right|^{2} d x d t+2 \beta_{5} \int_{Q_{\tau}}\left|\nabla c_{i}^{(k+1)}\right|^{2} d x d t, \quad i=1,2, \quad
\end{gathered}
$$


and

$$
\begin{gathered}
\left|\int_{0}^{\tau} \int_{\Gamma_{N}} g_{i}^{(k)}\left(c_{i}^{(k+1)}-1\right) d S_{x} d t\right| \leqslant \int_{0}^{\tau} \int_{\Gamma_{N}}\left|g_{i}^{(k)} c_{i}^{(k+1)}\right| d S_{x} d t+\int_{0}^{\tau} \int_{\Gamma_{N}}\left|g_{i}^{(k)}\right| d S_{x} d t \\
\quad \leqslant \frac{1}{2} \tau\left|\Gamma_{N}\right|+\left(\frac{1}{2 \beta_{6}}+\frac{1}{2}\right) \int_{0}^{\tau} \int_{\Gamma_{N}}\left|g_{i}^{(k)}\right|^{2} d S_{x} d t+\frac{\beta_{6} K_{0}}{2}\left\|c_{i}^{(k+1)}\right\|_{L^{2}\left(0, \tau ; H^{1}(\Omega)\right)}^{2},
\end{gathered}
$$

where $K_{0}$ is the constant from the trace theorem (3.1).

With the help of assumptions (2.7a) applied to the boundary terms $g_{i}^{(k)}$ and the obtained estimate $(3.12)$ of $\varphi^{(k+1)}$ we estimate the boundary term in the righthand side of (3.15) from above:

$$
\int_{0}^{\tau} \int_{\Gamma_{N}}\left|g_{i}^{(k)}\right|^{2} d S_{x} d t \leqslant\left(\beta_{1}+\beta_{2}\left\|\varphi^{(k+1)}\right\|_{L^{2}\left(0, T ; H^{1}(\Omega)\right)}^{2}\right) G\left(\mathbf{c}^{(k)}\right) \leqslant \beta_{1}+\beta_{2} K_{\varphi} \tau .
$$

Summing up (3.14) and (3.15), using the following integration

$$
\int_{Q_{\tau}} \frac{\partial}{\partial t}\left(c_{i}^{(k+1)}-1\right)^{2} d x d t=\int_{\Omega}\left(c_{i}^{(k+1)}(\tau)-1\right)^{2} d x-\int_{\Omega}\left(c_{i}^{0}-1\right)^{2} d x,
$$

and (3.12), from (3.13) it follows the inequalities below for $i=1,2$ :

$$
\begin{aligned}
& \frac{1}{2} \int_{\Omega}\left(c_{i}^{(k+1)}(\tau)-1\right)^{2} d x+\int_{Q_{\tau}}\left|\nabla c_{i}^{(k+1)}\right|^{2} d x d t \\
& \leqslant \frac{1}{2} \tau\left|\Gamma_{N}\right|+\left(\frac{1}{2 \beta_{6}}+\frac{1}{2}\right)\left(\beta_{1}+\beta_{2} K_{\varphi} \tau\right)+\frac{\beta_{6} K_{0}}{2}\left\|c_{i}^{(k+1)}\right\|_{L^{2}\left(0, \tau ; H^{1}(\Omega)\right)}^{2} \\
&+\frac{2}{\beta_{5}} K_{\varphi} \tau+2 \beta_{5} \int_{Q_{\tau}}\left|\nabla c_{i}^{(k+1)}\right|^{2} d x d t+\frac{1}{2} \int_{\Omega}\left|c_{i}^{0}-1\right|^{2} d x .
\end{aligned}
$$

Therefore, using the Poincaré inequality (3.3), we continue (3.17) further for $i=$ 1, 2:

$$
\begin{aligned}
& \frac{1}{2} \int_{\Omega}\left(c_{i}^{(k+1)}(\tau)-1\right)^{2} d x+k_{2}|| c_{i}^{(k+1)} \|_{L^{2}\left(0, \tau ; H^{1}(\Omega)\right)}^{2} \\
& \quad \leqslant \frac{1}{2} \tau\left|\Gamma_{N}\right|+\frac{1}{2}\left(\frac{1}{\beta_{6}}+1\right)\left(\beta_{1}+\beta_{2} K_{\varphi} \tau\right)+\frac{2}{\beta_{5}} K_{\varphi} \tau+\frac{1}{2} \int_{\Omega}\left|c_{i}^{0}-1\right|^{2} d x .
\end{aligned}
$$

In the left-hand side of (3.18), the constant $k_{2}$ is positive:

$$
k_{2}:=\left(\frac{1}{1+K_{P}}-\frac{\beta_{6} K_{0}}{2}-2 \beta_{5}\right)>\frac{1}{2\left(1+K_{P}\right)}>0
$$

for $\beta_{5}$ and $\beta_{6}$ chosen sufficiently small.

For convenience, we define the function space $\mathcal{W}$ equipped with the norm

$$
\|\mid \mathbf{c}\| \|^{2}:=\sum_{i=1}^{2}\left(\left\|c_{i}\right\|_{L^{\infty}\left(0, T ; L^{2}(\Omega)\right)}^{2}+\frac{1}{1+K_{P}}\left\|c_{i}\right\|_{L^{2}\left(0, T ; H^{1}(\Omega)\right)}^{2}\right) .
$$


Then, after summation over $i=1,2$ and taking the supremum over $\tau \in(0, T)$ in (3.18), we get:

$$
\left\|\mid \mathbf{c}^{(k+1)}-1\right\| \|^{2} \leqslant \bar{K}_{c}+\bar{\gamma}_{c} K_{\varphi}
$$

where $\quad \bar{K}_{c}:=2 T\left|\Gamma_{N}\right|+\frac{2 \beta_{1}}{\beta_{6}}+\sum_{i=1}^{2} \int_{\Omega}\left|c_{i}^{0}-1\right|^{2} d x \quad$ and $\quad \bar{\gamma}_{c}:=2 T\left(\frac{\beta_{2}}{\beta_{6}}+\frac{4}{\beta_{5}}\right)$.

Consequently, applying the triangle inequality $\left\|\left|\mathbf{c}^{(k+1)}\left\|\left.\right|^{2}=\right\|\left\|\mathbf{c}^{(k+1)}-1+1\right\| \|^{2} \leqslant\right.\right.$ $2\left|\left\|\mathbf{c}^{(k+1)}-1|\||^{2}+4\left(|\Omega|+\frac{\left|Q_{T}\right|}{1+K_{P}}\right)\right.\right.$, we have the estimate for $\mathbf{c}^{(k+1)}$ :

$$
\left\|\mathbf{c}^{(k+1)}\right\| \|^{2} \leqslant K_{c}+\gamma_{c} K_{\varphi}
$$

where $K_{c}:=4\left(|\Omega|+\frac{\left|Q_{T}\right|}{1+K_{P}}\right)+2 \bar{K}_{c}$ and $\gamma_{c}:=2 \bar{\gamma}_{c} K_{\varphi}$.

The mapping $\mathfrak{M}:\left(\mathbf{c}^{(k)}, \varphi^{(k)}\right) \mapsto\left(\mathbf{c}^{(k+1)}, \varphi^{(k+1)}\right)$ defined by (3.4) and (3.5) is continuous and maps the following set into itself:

$$
\begin{aligned}
S:= & \left\{(\mathbf{c}, \varphi) \in \mathcal{W} \times L^{\infty}\left(0, T ; H^{1}(\Omega)\right):\right. \\
& \left.\|\mathbf{c}\|\left\|^{2} \leqslant K_{c}+\gamma_{c} K_{\varphi},\right\| \varphi\left\|_{L^{\infty}\left(0, T ; H^{1}(\Omega)\right)}^{2}+\right\| \varphi \|_{L^{\infty}\left(0, T ; L^{2}\left(\Gamma_{N}\right)\right)}^{2} \leqslant K_{\varphi}\right\} .
\end{aligned}
$$

Therefore, the existence of a fixed point of $\mathfrak{M}$ follows by the Schauder-Tikhonov theorem. The proof is completed.

In order to get the existence of the solution of the complete formulation, we show the equivalence of the complete and the reduced formulations under the assumptions (2.7b) and (2.7c). For this purpose we consider two auxiliary lemmas.

Lemma 3.2 (Volume conservation). Under the assumption (2.7b) holding at the boundary, the volume balance $c_{1}+c_{2}=2$ is satisfied a.e. in $Q_{T}$.

Proof. We denote the difference by $\sigma:=c_{1}+c_{2}-2$, and find that $\left.\sigma\right|_{t=0}=c_{1}^{0}+$ $c_{2}^{0}-2=0$. By summing up (2.18a) over $i=1,2$ tested with the function $\sigma$, due to the mass conservation $(2.7 \mathrm{~b})$, it follows

$$
\int_{Q_{\tau}} \frac{\partial \sigma}{\partial t} \sigma d x d t+\int_{Q_{\tau}}|\nabla \sigma|^{2} d x d t=0
$$

We integrate the first term in (3.22) over $t \in(0, \tau)$ and obtain

$$
\left.\frac{1}{2} \int_{\Omega} \sigma^{2}\right|_{t=\tau} d x+\int_{Q_{\tau}}|\nabla \sigma|^{2} d x d t=\left.\frac{1}{2} \int_{\Omega} \sigma^{2}\right|_{t=0} d x
$$

Since $\left.\sigma\right|_{t=0}=0$, we conclude that $\sigma \equiv 0$, then $c_{1}+c_{2} \equiv 2$, thus proving the assertion.

Lemma 3.3 (Weak maximum principle). If the boundary terms $g_{1}(\mathbf{c}, \varphi)$ and $g_{2}(\mathbf{c}, \varphi)$ satisfy the assumption $(2.7 \mathrm{c})$, then the solution $\mathbf{c} \geqslant 0$ is non-negative a.e. in $Q_{T}$ for all $T$. For small $T$, the solution $\mathbf{c}>0$ is positive by continuity. 
Proof. Inserting the test function $\bar{c}_{i}=-c_{i}^{-}$for $i=1,2$ in (2.18a) because $c_{i}^{-}=0$ at $(0, T) \times \Gamma_{D}$ due to $(2.4)$, and using the decomposition $c_{i}=c_{i}^{+}-c_{i}^{-}$, we get

$$
\begin{aligned}
\int_{Q_{\tau}} \frac{\partial\left(c_{i}^{+}-c_{i}^{-}\right)}{\partial t}\left(-c_{i}^{-}\right) d x d t+\int_{Q_{\tau}}\left(\nabla\left(c_{i}^{+}-c_{i}^{-}\right)+\Upsilon\left(c_{i}^{+}\right) \nabla \varphi\right) \nabla\left(-c_{i}^{-}\right) d x d t \\
=\int_{0}^{\tau} \int_{\Gamma_{N}} g_{i}(\mathbf{c}, \varphi)\left(-c_{i}^{-}\right) d S_{x} d t, \quad i=1,2 .
\end{aligned}
$$

After application of the pointwise orthogonality $c_{i}^{-} c_{i}^{+}=0$, this results in the following equality:

$$
\frac{1}{2} \int_{Q_{\tau}} \frac{\partial\left(c_{i}^{-}\right)^{2}}{\partial t} d x d t+\int_{Q_{\tau}}\left|\nabla c_{i}^{-}\right|^{2} d x d t=-\int_{0}^{\tau} \int_{\Gamma_{N}} g_{i}(\mathbf{c}, \varphi) c_{i}^{-} d S_{x} d t, \quad i=1,2 .
$$

Since $g_{i}(\mathbf{c}, \varphi) c_{i}^{-}=0$ by assumption $(2.7 \mathrm{c})$ and using the following integration by parts $\frac{1}{2} \int_{Q_{\tau}} \frac{\partial\left(c_{i}^{-}\right)^{2}}{\partial t} d x d t=\left.\frac{1}{2} \int_{\Omega}\left(c_{i}^{-}\right)^{2} d x\right|_{0} ^{\tau}$, we have

$$
\frac{1}{2} \int_{\Omega}\left(c_{i}^{-}(\tau)\right)^{2} d x+\int_{Q_{\tau}}\left|\nabla c_{i}^{-}\right|^{2} d x d t=\frac{1}{2} \int_{\Omega}\left(\left(c_{i}^{0}\right)^{-}\right)^{2} d x, \quad i=1,2 .
$$

After summation over $i=1,2$ and taking the supremum over $\tau \in(0, T)$ in (3.23), with the help of Poincaré inequality (3.3) we conclude:

$$
\begin{aligned}
\left.\frac{1}{2}\left\|\mathbf{c}^{-}\right\|\right|^{2} \leqslant \frac{1}{2}\left\|\mathbf{c}^{-}\right\|_{L^{\infty}\left(0, T ; L^{2}(\Omega)\right)}^{2}+\frac{1}{1+K_{P}}\left\|\mathbf{c}^{-}\right\|_{L^{2}\left(0, T ; H^{1}(\Omega)\right)}^{2} & \\
& \leqslant\left\|\left(\mathbf{c}^{0}\right)^{-}\right\|_{L^{2}(\Omega)}^{2} .
\end{aligned}
$$

Since $\mathbf{c}^{0}>0$ according to $(2.9 \mathrm{~b})$, it follows $\mathbf{c}^{-}=0$ a.e. on $Q_{T}$. Then $\mathbf{c}=\mathbf{c}^{+}$, thus the solution is non-negative a.e. in $Q_{T}$. The lemma is proved.

If Lemmas 3.2 and 3.3 hold, then the solution of the reduced problem satisfies the constraint (2.6) and solves the complete problem. Rigorously this fact will be proved in the following

Lemma 3.4 (Equivalence of two formulations). Under assumptions of Lemma 3.2 and Lemma 3.3, the complete problem and the reduced problem are equivalent.

Proof. First, we show that the equation (2.11) and the conditions (2.6) lead to (2.18). Due to (2.6) we have

$$
c_{1}=c_{1}^{+}, \quad c_{2}=c_{2}^{+}, \quad \text { and } \quad c_{1}^{+}+c_{2}^{+}=2 .
$$

Therefore, from $(2.11 \mathrm{~b})$ we obtain $(2.18 \mathrm{~b})$ by using $(3.25)$, which provides that:

$$
\frac{1}{2}\left(c_{1}-c_{2}\right)=\frac{c_{1}^{+}-c_{2}^{+}}{c_{1}^{+}+c_{2}^{+}}=\Upsilon\left(\mathbf{c}^{+}\right)
$$


Using (3.25) again, after substitution of the expressions (2.1c) and (2.1d) in $\nabla \mu_{1}$ and $\nabla \mu_{2}$, with the help of (2.16) we can rewrite the following expressions equivalently:

$$
\begin{aligned}
& c_{1} \nabla \mu_{1}=c_{1}\left(\frac{\nabla c_{1}}{c_{1}}+\nabla p+\nabla \varphi\right)=\nabla c_{1}+c_{1} c_{2} \nabla \varphi=\nabla c_{1}+\Upsilon_{1}\left(\mathbf{c}^{+}\right) \nabla \varphi, \\
& c_{2} \nabla \mu_{2}=c_{2}\left(\frac{\nabla c_{2}}{c_{2}}+\nabla p-\nabla \varphi\right)=\nabla c_{2}-c_{2} c_{1} \nabla \varphi=\nabla c_{2}+\Upsilon_{2}\left(\mathbf{c}^{+}\right) \nabla \varphi .
\end{aligned}
$$

Consequently, from (2.11a) we arrive at equations (2.18a).

In return, if (2.18) holds, then it follows (2.11) and (2.6). Indeed, from Lemma 3.3 we have $c_{1}>0$ and $c_{2}>0$, and from Lemma 3.2 we get $c_{1}+c_{2}=2$ a.e. in $Q_{T}$, thus (2.6). This implies equalities (3.26) and (3.27), hence (2.11) holds. This proves the assertion of the lemma.

As a consequence of Lemma 3.4, it follows

Theorem 3.5 (Well-posedness of generalized Poisson-Nernst-Planck system). Let assumptions (2.7) on the boundary data hold. Then there exists a weak solution of the problem (2.11), satisfying a-priori estimates:

$$
\begin{gathered}
\|\varphi\|_{L^{\infty}\left(0, T ; H^{1}(\Omega)\right)}^{2}+\|\varphi\|_{L^{\infty}\left(0, T ; L^{2}\left(\Gamma_{N}\right)\right)}^{2} \leqslant K_{\varphi}, \\
\|\mathbf{c}\|_{L^{\infty}\left(0, T ; L^{2}(\Omega)\right)}^{2}+\|\mathbf{c}\|_{L^{2}\left(0, T ; H^{1}(\Omega)\right)}^{2} \leqslant K_{c}+\gamma_{c} K_{\varphi}
\end{gathered}
$$

with the positive constants $K_{\varphi}$ from (3.12), $\gamma_{c}$ and $K_{c}$ from (3.21).

Proof. By Lemma 3.1 there exists a weak solution of the reduced problem (2.18). Then by Lemma 3.4 it solves the complete problem (2.11), and estimates (3.12) and (3.21) hold true for the solution of the complete formulation, too.

\subsection{The uniqueness of the solution}

We emphasize that uniqueness of solutions of nonlinear systems cannot be proved generally. In fact, to prove the uniqueness of the solution of the underlying problem, additional smoothness of $\varphi$ and smallness of $g_{1}(\mathbf{c}, \varphi), g_{2}(\mathbf{c}, \varphi)$ are claimed.

Theorem 3.6 (Uniqueness). Let the solution component $\varphi$ be smooth such that

$$
\varphi \in L^{\infty}\left(Q_{T}\right), \nabla \varphi \in L^{\infty}\left(Q_{T}\right)^{d},
$$

and let there exist $0<K_{g}(\mathbf{c}, \varphi, \nabla \varphi)<\beta_{7}$ and $0<\delta<1$ :

$$
\int_{\Gamma_{N}} g_{i}(\mathbf{c}, \varphi) \bar{c} d S_{x} \leqslant \frac{K_{g}(\mathbf{c}, \varphi, \nabla \varphi)}{\beta_{7}} \int_{\Omega} \bar{c}^{2} d x+\beta_{7} \int_{\Omega}|\nabla \bar{c}|^{2} d x, \quad i=1,2,
$$

for all $\bar{c}$, c and for all $\beta_{7} \in(0, \delta)$. Then the weak solution (2.10) of the problem (2.11) with the boundary (2.4) and initial (2.5) conditions is unique. 
Proof. Let us assume that there exist two different solutions $\left(c_{1}^{(1)}, c_{2}^{(1)}, \varphi^{(1)}\right)$ and $\left(c_{1}^{(2)}, c_{2}^{(2)}, \varphi^{(2)}\right)$ of the problem (2.18) marked by indexes $m=1,2$ :

$$
\begin{aligned}
& \int_{\Omega} \frac{\partial c_{i}^{(m)}}{\partial t} \bar{c}_{i} d x+\int_{\Omega} \Upsilon_{i}\left(\mathbf{c}^{(m)}\right) \nabla \bar{c}_{i} d x \\
&=\int_{\Gamma_{N}} g_{i}\left(\mathbf{c}^{(m)}, \varphi^{(m)}\right) \bar{c}_{i} d S_{x}, \quad i=1,2 ; \\
& \int_{\Omega} \nabla\left(\varphi^{(m)}\right)^{\top} \nabla \bar{\varphi} d x+\int_{\Gamma_{N}} \varphi^{(m)} \bar{\varphi} d S_{x}=\int_{\Gamma_{N}} g \bar{\varphi} d S_{x}+\int_{\Omega} \Upsilon\left(\mathbf{c}^{(m)}\right) \bar{\varphi} d x
\end{aligned}
$$

where (3.32) is obtained from (2.18a) by differentiation with respect to $\tau$. In the following, the difference between these solutions will be denoted by $\tilde{\varphi}=\varphi^{(1)}-\varphi^{(2)}$, $\tilde{c}_{i}=c_{i}^{(1)}-c_{i}^{(2)}$, and $\tilde{g}_{i}=g_{i}\left(\mathbf{c}^{(1)}, \varphi^{(1)}\right)-g_{i}\left(\mathbf{c}^{(2)}, \varphi^{(2)}\right)$ for $i=1,2$.

Estimation for $\tilde{\varphi}$. Subtracting the equations (3.33) for $m=1$ and $m=2$ and substituting the test function $\tilde{\varphi}$, we obtain

$$
\int_{\Omega}|\nabla \tilde{\varphi}|^{2} d x+\int_{\Gamma_{N}} \tilde{\varphi}^{2} d S_{x}=\int_{\Omega}\left(\Upsilon\left(\mathbf{c}^{(1)}\right)-\Upsilon\left(\mathbf{c}^{(2)}\right)\right) \tilde{\varphi} d x
$$

Applying to (3.34) Young's inequality with a constant $\beta_{8}>0$ and the Poincaré inequality (3.2), it follows the upper bound

$$
\begin{aligned}
\left|\int_{\Omega}\left(\Upsilon\left(\mathbf{c}^{(1)}\right)-\Upsilon\left(\mathbf{c}^{(2)}\right)\right) \tilde{\varphi} d x\right| \leqslant & \frac{1}{2} \int_{\Omega}\left|\tilde{c}_{1}-\tilde{c}_{2}\right||\tilde{\varphi}| d x \\
& \leqslant \frac{1}{4 \beta_{8}} \sum_{i=1}^{2} \int_{\Omega} \tilde{c}_{i}^{2} d x+\frac{\beta_{8} K_{0}}{4} \int_{\Omega}|\nabla \tilde{\varphi}|^{2} d x .
\end{aligned}
$$

Henceforth, from the above estimates (3.34) and (3.35) we derive

$$
\left(\frac{1}{1+K_{P}}-\frac{\beta_{8} K_{0}}{4}\right)\|\nabla \tilde{\varphi}\|_{L^{2}(\Omega)}^{2}+\|\tilde{\varphi}\|_{L^{2}\left(\Gamma_{N}\right)}^{2} \leqslant \frac{1}{4 \beta_{8}} \sum_{i=1}^{2} \int_{\Omega} \tilde{c}_{i}^{2} d x
$$

and for small $\beta_{8}$ we get equivalently

$$
\|\nabla \tilde{\varphi}\|_{L^{2}(\Omega)}^{2}+\|\tilde{\varphi}\|_{L^{2}\left(\Gamma_{N}\right)}^{2} \leqslant \tilde{K}_{\varphi} \sum_{i=1}^{2}\left\|\tilde{c}_{i}\right\|_{L^{2}(\Omega)}^{2},
$$

with the positive factor

$$
\tilde{K}_{\varphi}:=\frac{1}{4 \beta_{8} \min \left\{1, \frac{1}{1+K_{P}}-\frac{\beta_{8} K_{0}}{4}\right\}}>0 .
$$


Estimation for $\tilde{\mathbf{c}}$. Next, we consider the difference between two solutions of (3.32) with the test function $\tilde{c}_{i}$ and sum it over $i=1,2$ to obtain

$$
\begin{aligned}
& \sum_{i=1}^{2}\left\{\frac{d}{d t}\left[\frac{1}{2} \int_{\Omega} \tilde{c}_{i}(t)^{2} d x\right]+\int_{\Omega}\left|\nabla \tilde{c}_{i}(t)\right|^{2} d x\right\}=\sum_{i=1}^{2}\left\{I_{1}^{i}+I_{2}^{i}\right\}, \\
& I_{1}^{i}:=\int_{\Gamma_{N}} \tilde{g}_{i} \tilde{c}_{i} d S_{x}, \quad I_{2}^{i}:=-\int_{\Omega}\left(\Upsilon_{i}\left(\mathbf{c}^{(1)}\right) \nabla \varphi^{(1)}-\Upsilon_{i}\left(\mathbf{c}^{(2)}\right) \nabla \varphi^{(2)}\right)^{\top} \nabla \tilde{c}_{i} d x .
\end{aligned}
$$

According to the assumption $(3.31)$ on $g_{i}(\mathbf{c}, \varphi)$ we estimate $I_{1}^{i}$ :

$$
\left|I_{1}^{i}\right|=\left|\int_{\Gamma_{N}} \tilde{g}_{i} \tilde{c}_{i} d S_{x}\right| \leqslant \frac{K_{g}}{\beta_{7}} \int_{\Omega} \tilde{c}_{i}^{2} d x+\beta_{7} \int_{\Omega}\left|\nabla \tilde{c}_{i}\right|^{2} d x .
$$

On the other hand, the integral $I_{2}^{i}$ can be rewritten equivalently as

$$
\begin{aligned}
I_{2}^{i}=-\int_{\Omega} z_{i}\left(c_{1}^{(1)} c_{2}^{(1)} \nabla \varphi^{(1)}-c_{1}^{(2)} c_{2}^{(1)} \nabla \varphi^{(1)}+c_{1}^{(2)} c_{2}^{(1)} \nabla \varphi^{(1)}-c_{1}^{(2)} c_{2}^{(2)} \nabla \varphi^{(1)}\right. \\
\left.+c_{1}^{(2)} c_{2}^{(2)} \nabla \varphi^{(1)}-c_{1}^{(2)} c_{2}^{(2)} \nabla \varphi^{(2)}\right)^{\top} \nabla \tilde{c}_{i} d x
\end{aligned}
$$

and estimated using Young's inequality with $\beta_{9}>0$ :

$$
\left|I_{2}^{i}\right| \leqslant\left|\int_{\Omega} z_{i}\left(\tilde{c}_{1} c_{2}^{(1)} \nabla \varphi^{(1)}+c_{1}^{(2)} \tilde{c}_{2} \nabla \varphi^{(1)}+c_{1}^{(2)} c_{2}^{(2)} \nabla \tilde{\varphi}\right)^{\top} \nabla \tilde{c}_{i} d x\right| .
$$

Then, applying Cauchy-Schwarz inequality, it follows:

$$
\begin{aligned}
\left|I_{2}^{i}\right| \leqslant \frac{\beta_{9}}{2}\left\|\nabla \tilde{c}_{i}\right\|_{L^{2}(\Omega)}^{2} & +\frac{1}{2 \beta_{9}}\left\{\left\|\tilde{c}_{1}\right\|_{L^{2}(\Omega)}^{2}\left\|c_{2}^{(1)}\right\|_{L^{\infty}(\Omega)}^{2}\left\|\nabla \varphi^{(1)}\right\|_{L^{\infty}(\Omega)}^{2}\right. \\
+ & \left.\left\|c_{1}^{(2)}\right\|\right|_{L^{\infty}(\Omega)} ^{2}\left\|\tilde{c}_{2}\right\|_{L^{2}(\Omega)}^{2}\left\|\nabla \varphi^{(1)}\right\| \|_{L^{\infty}(\Omega)}^{2} \\
& \left.+\left\|c_{1}^{(2)}\right\|_{L^{\infty}(\Omega)}^{2}\left\|c_{2}^{(2)}\right\| L_{L^{\infty}(\Omega)}^{2}\|\nabla \tilde{\varphi}\|_{L^{2}(\Omega)}^{2}\right\} .
\end{aligned}
$$

The assumption (3.30) implies that there exists $K_{3}>0$ such that $\left\|\nabla \varphi^{(1)}\right\|_{L^{\infty}(\Omega)}^{2} \leqslant$ $K_{3}$. Taking conditions (2.6) into account, we have $\left\|c_{1}^{(2)}\right\|_{L^{\infty}(\Omega)}^{2}<2,\left\|c_{2}^{(1)}\right\|_{L^{\infty}(\Omega)}^{2}<$ 2 and $\left\|c_{2}^{(2)}\right\|_{L^{\infty}(\Omega)}^{2}<2$. Denoting by $\gamma:=\left(\left(18 / \beta_{9}\right)\right) \max \left\{K_{2}, 4 \tilde{K}_{\varphi}, K_{g} / \beta_{7}\right\}$, and with the help of (3.37), it follows

$$
\begin{aligned}
\sum_{i=1}^{2}\left\{\frac{d}{d t}\left[\int_{\Omega} \tilde{c}_{i}(t)^{2} d x\right]+2\left(1-\beta_{7}-\beta_{9}\right)\left\|\nabla \tilde{c}_{i}(t)\right\|_{L^{2}(\Omega)}^{2}\right\} & \\
& \leqslant \gamma \sum_{i=1}^{2}\left\|\tilde{c}_{i}(t)\right\|_{L^{2}(\Omega)}^{2} .
\end{aligned}
$$


Therefore, choosing $\beta_{7}, \beta_{9}$ sufficiently small such that $1-\beta_{7}-\beta_{9}>0$, from (3.38) we infer

$$
\frac{d}{d t} \sum_{i=1}^{2}\left\|\tilde{c}_{i}(t)\right\|_{L^{2}(\Omega)}^{2} \leqslant \gamma \sum_{i=1}^{2}\left\|\tilde{c}_{i}(t)\right\|_{L^{2}(\Omega)}^{2} .
$$

Finally, from (3.39) by Grönwall's inequality we have

$$
\sum_{i=1}^{2}\left\|\tilde{c}_{i}(t)\right\|_{L^{2}(\Omega)}^{2} \leqslant \sum_{i=1}^{2}\left\|\tilde{c}_{i}(0)\right\|_{L^{2}(\Omega)}^{2} e^{\gamma t}=0,
$$

since $\tilde{c}_{i}(0)=0$. This implies that $\tilde{c}_{1} \equiv 0$ and $\tilde{c}_{2} \equiv 0$. Henceforth, $\tilde{\varphi} \equiv 0$ in (3.37) thus proving uniqueness of the solution and the assertion of the theorem.

\subsection{The Lyapunov stability and the entropy estimate}

For positive concentrations we can define a Lyapunov function:

$$
S: \mathbb{R}^{+} \rightarrow \mathbb{R}^{+}, \quad S(t):=\sum_{i=1}^{2} \int_{\Omega} c_{i} \ln c_{i} d x
$$

which associates the entropy. This function is non-negative, since $\xi \ln \xi \geqslant \xi-1$ for all $\xi>0$ and using the mass conservation (2.6a) it follows:

$$
S=\sum_{i=1}^{2} \int_{\Omega} c_{i} \ln c_{i} d x \geqslant \sum_{i=1}^{2}\left(c_{i}-1\right)=0 .
$$

The Lyapunov stability is characterized by the non-negativeness of the socalled dissipation function:

$$
D: \mathbb{R}^{+} \rightarrow \mathbb{R}, \quad D(t):=-\frac{d S}{d t}=-\sum_{i=1}^{2} \int_{\Omega} \frac{\partial c_{i}}{\partial t}\left(\ln c_{i}+1\right) d x
$$

which can be expressed with the help of the entropy estimate below.

Theorem 3.7 (Lyapunov stability). The entropy dissipation defined in (3.40) has the following equivalent expression:

$$
\begin{aligned}
& D=D_{1}+D_{2} \\
& D_{1}:=\sum_{i=1}^{2} \int_{\Omega}\left(2\left|\nabla\left(\sqrt{c_{i}}\right)\right|\right)^{2} d x+\int_{\Omega}\left(c_{1}-c_{2}\right)^{2} d x \\
& D_{2}:=\int_{\Gamma_{N}}(g-\varphi)\left(c_{1}-c_{2}\right) d S_{x}-\sum_{i=1}^{2} \int_{\Gamma_{N}} g_{i}(\mathbf{c}, \varphi) \ln c_{i} d S_{x} .
\end{aligned}
$$

By this, $D_{1}>0$ and the dissipation inequality $D \geqslant 0$ holds for $D_{2}$ either nonnegative or sufficiently small. 
Proof. We consider a quasi-stationary version of the system (2.11) written without integration over time, which depends on $t \in(0, T]$ as a parameter. Let $t$ be fixed.

Choosing the test function $\bar{c}_{i}=\ln c_{i}$, since $\ln c_{i}=\ln 1=0$ at the boundary $(0, T) \times \Gamma_{D}$, using the equation for the pressure (2.1c) and for electrostatic potentials $(2.1 \mathrm{~d})$, it follows from (2.11a) that:

$$
\int_{\Omega} \frac{\partial c_{i}}{\partial t} \ln c_{i} d x+\int_{\Omega}\left(\nabla c_{i}+\Upsilon_{i}(\mathbf{c}) \nabla \varphi\right) \frac{\nabla c_{i}}{c_{i}} d x=\int_{\Gamma_{N}} g_{i}(\mathbf{c}, \varphi) \ln c_{i} d S_{x}
$$

After summation of (3.42) over $i=1,2$, and inserting the first expression from (2.15) here, we obtain

$$
\begin{aligned}
\sum_{i=1}^{2} \int_{\Omega} \frac{\partial c_{i}}{\partial t} \ln c_{i} d x+\sum_{i=1}^{2} \int_{\Omega} \frac{\left|\nabla c_{i}\right|^{2}}{c_{i}} d x+\int_{\Omega} \nabla \varphi^{\top} \nabla\left(c_{1}-c_{2}\right) d x & \\
& =\sum_{i=1}^{2} \int_{\Gamma_{N}} g_{i}(\mathbf{c}, \varphi) \ln c_{i} d S_{x} .
\end{aligned}
$$

In (3.43) the volume balance $(2.6 \mathrm{a}),(2.16)$, and the following transformation were used:

$$
\begin{aligned}
\sum_{i=1}^{2} \Upsilon_{i}(\mathbf{c}) \frac{\nabla c_{i}}{c_{i}}=c_{2} \nabla c_{1}-c_{1} \nabla c_{2}= & \frac{1}{2} \\
& \left(2 \nabla c_{1}-c_{1} \nabla\left(c_{1}+c_{2}\right)\right) \\
& +\frac{1}{2}\left(c_{2} \nabla\left(c_{1}+c_{2}\right)-2 \nabla c_{2}\right)=\nabla\left(c_{1}-c_{2}\right) .
\end{aligned}
$$

On the other hand, from (2.11b) with the test function $\bar{\varphi}=c_{1}-c_{2}$, which is zero at $(0, T) \times \Gamma_{D}$, we have

$$
\int_{\Omega} \nabla \varphi^{\top} \nabla\left(c_{1}-c_{2}\right) d x-\frac{1}{2} \int_{\Omega}\left(c_{1}-c_{2}\right)^{2} d x=\int_{\Gamma_{N}}(g-\varphi)\left(c_{1}-c_{2}\right) d S_{x} .
$$

Subtracting (3.44) from (3.43), with the help of the identity $\frac{\left|\nabla c_{i}\right|^{2}}{c_{i}}=\left(2\left|\nabla\left(\sqrt{c_{i}}\right)\right|\right)^{2}$ we infer

$$
\begin{aligned}
\sum_{i=1}^{2} \int_{\Omega} \frac{\partial c_{i}}{\partial t} \ln c_{i} d x & +\sum_{i=1}^{2} \int_{\Omega}\left(2\left|\nabla\left(\sqrt{c_{i}}\right)\right|\right)^{2} d x+\int_{\Omega}\left(c_{1}-c_{2}\right)^{2} d x \\
& =\sum_{i=1}^{2} \int_{\Gamma_{N}} g_{i}(\mathbf{c}, \varphi) \ln c_{i} d S_{x}-\int_{\Gamma_{N}}(g-\varphi)\left(c_{1}-c_{2}\right) d S_{x} .
\end{aligned}
$$

Noting that $\sum_{i=1}^{2} \frac{\partial c_{i}}{\partial t}=\frac{\partial}{\partial t} \sum_{i=1}^{2} c_{i}=\sum_{i=1}^{2} \frac{\partial}{\partial t} 2=0$, it holds $\sum_{i=1}^{2} \int_{\Omega} \frac{\partial c_{i}}{\partial t} \ln c_{i} d x=$ $\sum_{i=1}^{2} \int_{\Omega} \frac{\partial c_{i}}{\partial t}\left(\ln c_{i}+1\right) d x=-D$, and (3.45) leads to the formula (3.41). This justifies the assertion of the theorem. 
Discussion. First, the two-component dimensionless model can be generalized to the multi-phase model following [12, 13]. Second, physical parameters and matrices should be introduced into the system. In the latter case we have to assume special conditions for coefficient matrices in order to prove existence of the solution. We can prove that the solution is non-negative only if rather strong assumptions hold. Namely, the coefficient matrices in the parabolic system for the vector $\mathbf{c}$ should be diagonal, such that the system is decoupled. Otherwise, under weaker assumptions the solution guarantees to be non-negative only locally in time for small $T>0$.

\section{References}

[1] G. Allaire, R. Brizzi, J.-F. Dufrêche, A. Mikelić, A. Piatnitski, Ion transport in porous media: derivation of the macroscopic equations using upscaling and properties of the effective coefficients. Comp. Geosci. 17 (2013), 479-495.

[2] K. Becker-Steinberger, S. Funken, M. Landstorfer, K. Urban, A mathematical model for all solid-state lithium-ion batteries. ECS Trans. 25 (2010), 285-296.

[3] M. Burger, B. Schlake, M-T. Wolfram, Nonlinear Poisson-Nernst-Planck equations for ion flux through confined geometries. Nonlinearity 25 (2012), 961-990.

[4] L. Desvillettes, K. Fellner, Duality and entropy methods for reversible reactiondiffusion equations with degenerate diffusion. Math. Meth. Appl. Sci. 38 (2015), 34323443.

[5] W. Dreyer, C. Guhlke, R. Müller, Modeling of electrochemical double layers in thermodynamic non-equilibrium. Phys. Chem. Chem. Phys., 17 (2015), 27176-27194.

[6] M. Efendiev, Evolution Equations Arising in the Modelling of Life Sciences. Internat. Ser. Numer. Math. 163, Birkhäuser/Springer, 2013.

[7] K. Fellner, V.A. Kovtunenko, A discontinuous Poisson-Boltzmann equation with interfacial transfer: homogenisation and residual error estimate. Appl. Anal. DOI: 10.1080/00036811.2015.1105962.

[8] K. Fellner, V.A. Kovtunenko, A singularly perturbed nonlinear Poisson-Boltzmann equation: uniform and super-asymptotic expansions. Math. Meth. Appl. Sci. 38 (2015), 3575-3586.

[9] J. Fuhrmann, Comparison and numerical treatment of generalized Nernst-Planck Models. Comput. Phys. Commun. 196 (2015), 166-178.

[10] A. Glitzky, A. Mielke, A gradient structure for systems coupling reaction-diffusion effects in bulk and interfaces. Z. Angew. Math. Phys. 64 (2013), 29-52.

[11] M. Herz, N. Ray, P. Knabner, Existence and uniqueness of a global weak solution of a Darcy-Nernst-Planck-Poisson system. GAMM-Mitt. 35 (2012), 191-208.

[12] V. A. Kovtunenko, Electro-kinetic structure model with interfacial reactions. In Proc. 7th ECCOMAS Thematic Conference on Smart Structures and Materials SMART 2015, A.L. Araújo, C.A. Mota Soares et al. eds., IDMEC: Lisbon (2015).

[13] V.A. Kovtunenko, A.V. Zubkova, On generalized Poisson-Nernst-Planck equations with inhomogeneous boundary conditions: a-priori estimates and stability. Math. Meth. Appl. Sci., DOI: 10.1002/mma.4140. 
[14] O. A. Ladyzhenskaya, The Boundary Value Problems of Mathematical Physics. Appl. Math. Sci. 49, Springer Verlag, 1985.

[15] A. Latz, J. Zausch, O. Iliev, Modeling of species and charge transport in Li-ion batteries based on non-equilibrium thermodynamics. Lecture Notes in Comput. Sci. 6046 (2011), 329-337.

[16] T. Roubíček, Incompressible ionized non-Newtonean fluid mixtures. SIAM J. Math. Anal. 39 (2007), 863-890.

[17] T. Roubíček, Incompressible ionized fluid mixtures: a non-Newtonian approach. IASME Trans. 2 (2005), 1190-1197.

[18] S. A. Sazhenkov, E. V. Sazhenkova, A. V. Zubkova, Small perturbations of two-phase fluid in pores: Effective macroscopic monophasic viscoelastic behavior. Sib. Èlektron. Mat. Izv. 11 (2014), 127-158.

[19] M. Schmuck, Modeling and deriving porous media Stokes-Poisson-Nernst-Planck equations by a multi-scale approach. Commun. Math. Sci. 9 (2011), 685-710.

\section{Acknowledgment}

A. Z. is partially supported by IGDK1754, and V. K. acknowledgements the partial support of NAWI Graz and he thanks R. Duduchava for his support of the visit of the Humboldt-Kolleg in Tbilisi, IWOTA 2015, and Batumi 2015 Meetings.

The authors thank the Austrian Academy of Sciences (OeAW) and OeAD Scientific \& Technological Cooperation (WTZ CZ 01/2016) for partial support.

Victor A. Kovtunenko

Institute for Mathematics and Scientific Computing

Karl-Franzens University of Graz, NAWI Graz

Heinrichstraße 36, 8010 Graz, Austria

and Lavrentyev Institute of Hydrodynamics,

Siberian Division of the Russia Academy of Sciences, 630090 Novosibirsk, Russia

e-mail: victor.kovtunenko@uni-graz.at

Anna V. Zubkova

Institute for Mathematics and Scientific Computing

Karl-Franzens University of Graz, NAWI Graz

Mozartgasse 14, 8010 Graz, Austria

e-mail: anna.zubkova@uni-graz.at 